\title{
Prise en Charge des Brûlures Graves de l'enfant en Réanimation à l'hôpital Universitaire de Parakou au Bénin
}

\author{
Tchaou Blaise Adelin, \\ Tchégnonsi N’venonfon Charles Frédéric, \\ Houndjè Cocou Yélinhan Patrice, \\ Avossèvou Childéric Affiémin,
}

Unité d'Enseignement, de Formation et de Recherche en AnesthésieRéanimation et en Médecine d'Urgence,

Centre Hospitalier Universitaire Départemental, Borgou, Alibori, Faculté de

Médecine Université de Parakou, Bénin

Doi: 10.19044/esj.2019.v15n3p199 URL:http://dx.doi.org/10.19044/esj.2019.v15n3p199

Resume

Objectif : Décrire les aspects sociodémographiques, cliniques, paracliniques, thérapeutiques et évolutifs des brûlures graves de l'enfant en réanimation polyvalente à l'hôpital universitaire de Parakou au Bénin. Patients et méthodes : C'est une étude longitudinale à visée descriptive et analytique avec recueil rétrospectif des données laquelle a été réalisée du $1^{\mathrm{er}}$ janvier 2012 au 31 décembre 2016. Dans le recueil des données étaient inclus les patients âgés de moins de 16 ans présentant une brûlure grave et admis en réanimation. Résultats : Durant la période d'étude, 2966 patients ont été hospitalisés en réanimation, parmi lesquels nous avons dénombré 122 cas de brûlures (4,52\%) et de façon spécifique nous avons colligé 49 cas de brûlures graves de l'enfant représentant $40,16 \%$ de l'ensemble des brûlés et 1,65\% des admissions. L'âge moyen était de 3,5 $\pm 2,7$ ans ( 2 mois et 12 ans) et une prédominance masculine a été notée $(57,1 \%)$. Le délai médian d'admission était de 2 heures. L'ensemble des brûlures étaient accidentelles et 93,9\% d'entre elles étaient domestiques. Le mécanisme de survenue était thermique dans $100 \%$ des cas. L'ébouillantement constituait $67,3 \%$ et les flammes $32,7 \%$ des cas. La surface corporelle brûlée (SCB) moyenne était de 30,0 $\pm 19,2 \%$ et les brûlures du $2^{\text {ème }}$ degré représentaient $95,9 \%$. Le traitement comprenait un pansement occlusif avec une pommade à base de Trolamine (Biafine ${ }^{\circledR}$ ), un remplissage vasculaire, l'administration d'antalgique, d'antibiotique et d'antianémique voire la transfusion. La durée moyenne d'hospitalisation était de $7 \pm 5$ jours avec des extrêmes de un et 20 jours. Des complications étaient 
survenues chez $73,5 \%$ des patients : anémie $(75,0 \%)$, choc hypovolémique $(33,3 \%)$ et infection locale $(22,2 \%)$. La mortalité était de $40,8 \%$ et était liée à l'anémie, au choc hypovolémique et au sepsis. Conclusion : La brûlure grave met en jeu le pronostic vital de l'enfant, raison pour laquelle la prévention primaire doit être de mise.

Mots clés: Brûlures graves, enfant, surface corporelle brulée, réanimation

\title{
Management of Severe Burns of Children at the Intensive Care of the University Teaching Hospital of Parakou in Benin
}

\author{
Tchaou Blaise Adelin, \\ Tchégnonsi N'venonfon Charles Frédéric, \\ Houndjè Cocou Yélinhan Patrice, \\ Avossèvou Childéric Affiémin,
}

Unité d'Enseignement, de Formation et de Recherche en Anesthésie-

Réanimation et en Médecine d'Urgence,

Centre Hospitalier Universitaire Départemental, Borgou, Alibori, Faculté de

Médecine Université de Parakou, Bénin

\begin{abstract}
Objective: To describe the socio-demographic, clinical, paraclinical, therapeutic, and evolutionary aspects of children with severe burns in the general purpose intensive care units at Parakou Teaching Hospital in Benin. Patients and Methods: A longitudinal study with descriptive and analytical purposes and a retrospective data collection was conducted from January $1 \mathrm{st}$ 2012 to December 31st 2016. The patients were under 16 years of age, with a severe burn, and were admitted to the intensive care units. Results: During the study period, 2966 patients have been hospitalized in the intensive care units including 122 burns cases (4.52\%). Specifically, we recruited 49 children with severe burns representing $40.16 \%$ of all the burn victims and $1.65 \%$ of those that were admitted. The mean age was $3.5 \pm 2.7$ years ( 2 months and 12 years) with a male predominance (57.1\%). The median time for admission was 2 hours. All the burns were unintentional and $93.9 \%$ of them were domestic. The mechanism leading to their onset was thermal in $100 \%$ of cases. Scalding represented $67.3 \%$ and flame $32.7 \%$ of the cases. The average body surface
\end{abstract}


burned $(\mathrm{BSB})$ was $30.0 \pm 19.2 \%$ and $95.9 \%$ which represented 2 nd degree burn. The treatment constituted an occlusive dressing using Trolamine (Biafine®), a vascular filling, analgesic, antibiotic and anti-anaemic administration or even a blood transfusion. The mean duration of hospitalization was $7 \pm 5$ days with the extremes of 1 and 20 days. Complications occurred in $73.5 \%$ of patients: anaemia $(75.0 \%)$, hypovolemic shock $(33.3 \%)$, and local infection $(22.2 \%)$. The mortality was $40.8 \%$ and was related to anaemia, hypovolemic shock, and sepsis. Conclusion: The severe burn is life-threatening for the children. Hence, primary prevention must be the rule.

Keywords: Severe burns, Children, Body surface burned, intensive care units

\section{Introduction}

Urgence médico-chirurgicale par excellence, la brûlure est définie par la perte de l'intégrité de la barrière cutanée touchant l'épiderme (brûlures superficielles), le derme et/ou les structures sous-jacentes à la membrane basale (brûlures profondes), pouvant aller jusqu'à la carbonisation musculoaponévrotique et osseuse.

La brûlure de l'enfant constitue une cause importante de morbidité et de mortalité d'origine accidentelle. Elle reste une urgence fréquente dans notre pratique quotidienne. L'insouciance du jeune enfant associée à l'ignorance du risque, voire l'imprudence familiale, les exposent à ces risques (Zahid et al., 2011). Les brûlures graves par feu représentent la sixième cause de décès parmi les enfants de 5-14 ans (OMS, 2008). En Afrique Subsaharienne, Hyder et al. (2012) ont rapporté dans une étude une fréquence allant de 25 à $68 \%$ chez les enfants de moins de 5 ans. Il s'agit donc une affection fréquente et grave mettant en jeu souvent le pronostic vital des victimes en l'absence d'une prise en charge adéquate et codifiée. Dans les pays à ressources économiques limitées, le faible pouvoir d'achat de la population et le plateau technique médical inapproprié sont à l'origine d'une mortalité élevée. L'accent est donc de plus en plus mis sur la prévention (OMS, 2008). À cet égard, l'objectif de cette étude était de décrire les aspects sociodémographiques, cliniques, paracliniques, thérapeutiques évolutifs des brûlures graves de l'enfant en réanimation à l'hôpital universitaire de Parakou au Bénin.

\section{Patients et méthodes}

\section{Cadre d'étude}

L'unité de réanimation de l'hôpital universitaire de Parakou a servi de cadre d'étude. Il s'agit d'une unité de réanimation polyvalente fonctionnelle 24 heures sur 24 comportant 13 lits. Elle constitue le cadre de traitement des brûlures graves dont la prise en charge est pluridisciplinaire, faisant intervenir 
les anesthésiste-réanimateurs, les chirurgiens, les pédiatres et les kinésithérapeutes. Le personnel soignant est constitué de 3 médecins anesthésistes-réanimateurs, d'infirmiers (ères) d'aides-soignants et d'agents d'entretien et à ceux-là s'ajoutent les collaborateurs extérieurs précédemment cités. Elle dispose de 4 moniteurs multiparamétriques et de 3 respirateurs de réanimation, tout cela en dehors de quelques produits de premières nécessités: kits de perfusion, solutés de remplissages (cristalloïdes, macromolécules) et drogues de réanimation (antalgiques, analgésiques, sympathomimétiques, vagolytiques, diurétiques et corticoïdes). Les kits d'urgence spécifiques, notamment celui des brûlés, sont servis par la pharmacie principale de l'hôpital.

\section{Méthodes d'étude}

Il s'est agi d'une étude longitudinale à visée descriptive et analytique avec recueil rétrospectif des données à partir des dossiers des enfants brûlés graves réalisée sur une période de cinq ans allant du $1^{\text {er }}$ janvier 2012 au 31 décembre 2016. Étaient inclus dans l'échantillon selon les recommandations de (Women's and Children's Hospital - Paediatric Burns Service Guidelines, 2018):

Les enfants brûlés âgés de moins de cinq ans et dont la surface corporelle brûlée était $<5 \%$ et/ou chez qui l'examen a noté une brûlure profonde.

$>$ Les enfants brûlés âgés de plus de cinq ans et dont la surface corporelle brûlée était $\geq 10 \%$.

$>$ Les enfants brûlés âgés de plus de cinq ans et dont la surface corporelle brûlée était $<10 \%$ et associés aux critères suivants :

- Atteinte d'une zone à risque fonctionnel (mains, pieds, face, périnée et plis de flexion)

- Signes de gravité (inhalation de fumée suspectée ou avérée, lésions circulaires)

- Pathologies associées (polytraumatisme, insuffisance respiratoire chronique, cardiopathie / coronaropathie (sévère et ou instable)

- Difficultés à mettre en œuvre un traitement ambulatoire (hyperalgésie, conditions de vie défavorables, impossibilité à se rendre à la consultation)

- Mécanisme lésionnel (brûlures électriques, brûlures chimiques par acide fluorhydrique ou phosphorique)

Nous avons exclus de l'étude les brulés graves dont les parents ont refusé l'hospitalisation et les brulés graves décédés à l'admission.

Les variables étudiées étaient : les données sociodémographiques (âge, sexe), les données cliniques (mode d'admission, délai d'admission, moyen de 
transport, agent causal, surface corporelle brûlée (SCB) calculée partir de la table de Lund and Browder), les données paracliniques (Numération Formule Sanguine, ionogramme sanguin, azotémie, créatininémie, protidémie, glycémie et Goutte épaisse et Densité parasitaire), les données thérapeutiques (soins locaux réalisés au bloc opératoire sous sédation, remplissage vasculaire, antibiothérapie, antianémique) et les données évolutives et pronostiques (durée d'hospitalisation, complications et mode de sortie avec deux modalités :vivant ou décédé). Les données ont été traitées avec les logiciels Epi info version 3.5.1 et Microsoft Excel Logiciel.

\section{Résultats}

\section{Données sociodémographiques}

Durant la période d'étude, 2966 patients ont été hospitalisés en réanimation, parmi lesquels nous avons dénombré 122 cas de brûlure $(4,11 \%)$ et de façon spécifique nous avons colligé 49 cas de brûlures graves de l'enfant représentant $40,16 \%$ de l'ensemble des brûlés et 1,65\% des admissions. L'âge moyen des patients était de 3,5 $\pm 2,7$ ans avec des extrêmes de 2 mois et 12 ans. Les patients âgés de moins de 5 ans représentaient 71,4\% $(n=35)$. Les patients de sexe masculin représentaient $57,1 \%$ (sex-ratio de 1,3). Le Tableau 1 montre la répartition des patients en fonction des tranches d'âge et du sexe.

Tableau 1. Répartition des patients en fonction des tranches d'âge (ans) et du sexe.

\section{Sexe}

\begin{tabular}{|c|c|c|c|c|c|c|}
\hline & \multicolumn{2}{|c|}{ Masculin } & \multicolumn{2}{|c|}{ Féminin } & \multirow[b]{2}{*}{ Total } & \multirow[b]{2}{*}{ Pourcentage } \\
\hline & Nombre & Pourcentage & Nombre & Pourcentage & & \\
\hline $0-3$ & 12 & 24,50 & 11 & 22,45 & 23 & 46,95 \\
\hline $3-6$ & 11 & 22,45 & 5 & 10,20 & 16 & 32,65 \\
\hline $6-9$ & 4 & 8,16 & 4 & 8,16 & 8 & 16,32 \\
\hline $9-15$ & 1 & 2,04 & 1 & 2,04 & 2 & 4,08 \\
\hline Total & 28 & 57,15 & 21 & 42,85 & 49 & 100,00 \\
\hline
\end{tabular}

\section{Données cliniques et paracliniques}

L'admission était directe dans $69,4 \%$ des cas $(n=34)$ contre 15 patients $(30,6 \%)$ référés d'un autre centre de santé. Le délai médian d'admission après l'accident était de 2 heures et $71,4 \%$ des patients avaient été admis avant 6 heures. Aucun patient n'a bénéficié d'un transport médicalisé. Quatre-vingt pourcent des patients $(\mathrm{n}=39)$ ont été transportés à moto, $13 \%(\mathrm{n}=6)$ d'entre eux ont été transportés par un véhicule de transport en commun, $5 \%(\mathrm{n}=3)$ par les sapeurs-pompiers et $2 \%(\mathrm{n}=1)$ par une ambulance sans assistance médicale. Dans notre série tous les cas de brûlures graves des enfants étaient survenus dans un contexte d'accident et la majorité s'est produite à domicile $(93,9 \%)$. Le mécanisme était thermique dans $100 \%$ des cas. Il s'agissait de l'ébouillantement (67,3\%) et les flammes (32,7\%). Pour les ébouillantés, l'eau était l'agent causal dans $49,0 \%$ des cas. Le trimestre (décembre à février) avait 
enregistré $34,7 \%$ de cas de brûlures. La surface corporelle brûlée moyenne était de 30,0\% $\pm 19,2 \%(10,0 \%$ à $85,0 \%)$. Les SCB déterminées dans notre série étaient supérieures à $10 \%$ chez tous les enfants. Les enfants brûlés au $2^{\text {ème }}$ degré représentaient $95,9 \%$. Le Tableau 2 montre la répartition des patients en fonction de la surface corporelle brulée (SCB).

Tableau 2. Répartition des patients en fonction de la surface corporelle brulée

\begin{tabular}{lcc}
\hline & Effectif & Pourcentage \\
\hline$[10-20[$ & 17 & 34,7 \\
{$[20-40[$} & 19 & 38,8 \\
{$[40-60[$} & 7 & 14,3 \\
$\geq 60$ & 6 & 12,2 \\
Total & 49 & 100,0 \\
\hline
\end{tabular}

Un bilan paraclinique a été réalisé chez 37 brûlés (75,5\%). Le Tableau 3 montre la répartition des patients en fonction des examens paracliniques réalisés.

Tableau 3. Répartition des patients en fonction des examens paracliniques réalisés.

\begin{tabular}{lcc}
\hline & Effectif & Pourcentage \\
\hline Groupe Sanguin et Rhésus & 20 & 40,8 \\
Numération Formule Sanguine & 35 & 71,4 \\
Ionogramme sanguin & 17 & 34,7 \\
Azotémie & 27 & 55,1 \\
Créatininémie & 27 & 55,1 \\
Glycémie & 16 & 32,6 \\
Protidémie & 5 & 10,2 \\
Goutte épaisse - Densité parasitaire & 14 & 28,6 \\
\hline
\end{tabular}

Le Tableau 4 montre la répartition des patients en fonction des résultats des examens réalisés.

Tableau 4. Répartition des patients en fonction des résultats des examens paracliniques réalisés.

\begin{tabular}{lcc}
\hline & Moyenne $( \pm \mathrm{ET})$ & {$[\mathrm{Min}-\mathrm{Max}]$} \\
\hline Taux d'hémoglobine & $11,9( \pm 3,7)$ & {$[4,1-21,8]$} \\
Hématocrite & $0,36( \pm 0,10)$ & {$[0,15-0,63]$} \\
Nombre de globules blancs & $14,7( \pm 8,2)$ & {$[4,1-41,2]$} \\
Nombre de plaquettes & $340( \pm 120)$ & {$[145-440]$} \\
Natrémie & $132,7( \pm 6,1)$ & {$[120,4-142]$} \\
Kaliémie & $3,4( \pm 0,7)$ & {$[2,20-5,82]$} \\
Chlorémie & $104,2( \pm 5,7)$ & {$[96,9-112]$} \\
Azotémie & $0,24( \pm 0,11)$ & {$[0,05-0,46]$} \\
Créatininémie & $8,51( \pm 2,66)$ & {$[4,25-11,24]$} \\
Glycémie & $0,98( \pm 0,35)$ & {$[0,66-1,71]$} \\
Protidémie & $40,06( \pm 15,00)$ & {$[36,01-60,4]$} \\
\hline
\end{tabular}




\section{Données thérapeutiques}

L'information quant aux soins sur les lieux de la brûlure a été renseignée dans $87,76 \%$ des cas $(n=43)$. Quatorze patients $(32,6 \%)$ avaient bénéficié d'un lavage à grande eau contre 23 patients $(53,5 \%)$ qui n'avaient reçu aucun soin avant l'admission. Le Tableau 5 présente la répartition des patients selon les soins administrés sur les lieux de la brûlure.

Tableau 5. Répartition des patients selon les soins administrés sur les lieux de la brûlure.

\begin{tabular}{lcc}
\hline & Effectif & Pourcentage \\
\hline Aucun soin & $\mathbf{2 3}$ & $\mathbf{5 3 , 5}$ \\
Lavage à grande eau & $\mathbf{1 4}$ & $\mathbf{3 2 , 6}$ \\
Pâte dentifrice & 2 & 4,6 \\
Miel & 2 & 4,6 \\
Produits traditionnel & 2 & 4,6 \\
Total & $\mathbf{4 3}$ & $\mathbf{1 0 0 , 0}$ \\
\hline
\end{tabular}

En milieu hospitalier, le traitement local a consisté en un nettoyage au sérum salé, à l'excision des phlyctènes et au pansement occlusif avec une pommade à base de trolamine (Biafine ${ }^{\circledR}$ ) ou à l'éosine aqueuse réalisée au bloc opératoire sous sédation à base de Midazolam $(0,05$ à $0,2 \mathrm{mg} / \mathrm{kg} /$ dose intraveineuse directe associé à de la Kétamine (1 à $2 \mathrm{mg} / \mathrm{kg} /$ dose intraveineuse en $1 \mathrm{~min}$ ). En réanimation la prise en charge associait un remplissage vasculaire, la transfusion de culot globulaire en cas d'anémie, de plasma frais congelé en cas d'hypoprotidémie et le paracétamol injectable comme antalgique dans la majorité des cas. L'antibiothérapie était systématique dans notre série en raison d'une part des conditions de ramassage, du risque supplémentaire d'infection nosocomiale et d'autre part du fait que les enfants brûlés étaient hospitalisés dans la même salle que d'autres patients. Le traitement antibiotique associait soit la Ceftriaxone (50 $\mathrm{mg} / \mathrm{kg} / 24$ heures) au Métronidazole (10 $\mathrm{mg} / \mathrm{kg}$ toutes les 8 heures), ou Amoxicilline + acide Clavulanique (100 mg/kg en 3 prises par jour). Le traitement par sérovaccination contre le tétanos avait été réalisé chez 32 enfants $(63,30 \%)$ n'ayant pas bénéficié d'une vaccination antitétanique antérieure. Nous n'avons enregistré aucun cas de réalisation d'escarrotomie, d'aponévrotomie, ou de greffes de peau. L'alimentation orale riche en protéines avait été débutée 24 à 48 heures après stabilisation de l'état hémodynamique et respiratoire.

\section{Données évolutives et pronostiques}

La durée moyenne de séjour en réanimation était de $7 \pm 5$ jours avec des extrêmes de un (01) et 20 jours. Des complications ont été observées chez 36 patients $(73,4 \%)$. Les principales complications étaient l'anémie (31/49: $63,26 \%)$, le choc hypovolémique (16/49: 32,65\%), infection locale (10/49: 
$20,41 \%)$, sepsis grave $(5 / 49: 10,20 \%)$, insuffisance rénale $(2 / 49: 4,08 \%)$ et dénutrition $(2 / 49: 4,08 \%)$. La mortalité était de 40,81\% (20/49) et 17 enfants $(34,69 \%)$ sont décédés au cours de la première semaine. Cette mortalité élevée était liée à trois complications majeures : anémie, choc hypovolémique et sepsis.

\section{Discussion}

\section{Données sociodémographiques}

La fréquence annuelle de cas de brûlure objectivée dans notre étude était comparable à celle obtenue dans l'étude de Khongwar en Inde qui était de 13 cas par an (Khongwar et al. 2016). Les enfants sont généralement les premières victimes des brûlures graves comme l'ont rapporté d'autres études (Kouamé et al., 2013 ; Oludiran et al.,2009 ; Zahid et al.,2011). La grande turbulence des enfants, leur curiosité, leur insouciance et parfois la négligence des parents en seraient la cause. Ces facteurs sont le témoin de pourcentage élevé des patients de sexe masculin objectivé dans notre série.

\section{Données cliniques et paracliniques}

Pour leur transport à l'hôpital, très peu de patients bénéficient d'un transport médicalisé dans les pays les moins avancés. Au Cameroun, Ewono et al., 2014, rapportent qu'aucun patient n'avait bénéficié d'un transport médicalisé dans son étude contrairement à ce qui est observé dans les pays développés comme par exemple la France, où l' obligation est faite pour toute brûlure grave de l'enfant en dehors de l'hôpital de faire l'objet d'un appel immédiat à la régulation du centre $15 \mathrm{du}$ SAMU pour avis et transport médicalisé vers un centre de traitement des brûlés (CTB) (Pallier et al., 2018). L'indisponibilité d'une offre de transport médicalisé amène les parents à recourir à des moyens de transport personnel.

Tous nos patients ont été victimes de brûlure thermique et dans $67,3 \%$ des cas il s'agissait de l'eau chaude. Ce constat a été fait par d'autres auteurs qui ont rapporté que les enfants sont le plus souvent victimes d'accidents domestiques du fait des liquides chauds (Zahid et al., 2011 ; Behzad et al., 2014). Ce taux élevé de brûlure thermique pose l'épineux problème de la responsabilité des parents en ce qui concerne la surveillance des enfants et la nécessité d'adopter des mesures de sécurité afin de limiter au maximum la survenue de tel accident. Au vue de ce constat la prévention primaire est de mise et certaines mesures de sécurité doivent être prises, à savoir :

- garder les enfants à l'écart de la cuisine et des lieux de cuisson des aliments, ainsi que d'autres endroits où il $\mathrm{y}$ a des risques de brûlures ;

- limiter l'intensité des flammes et mettre la protection de la fournaise autour des fours traditionnels ;

- promouvoir et encourager l'utilisation de l'électricité domestique ; 
- éviter le stockage à domicile et la vente informelle d'hydrocarbure ;

- interdire formellement la vente d'essence falsifiée et augmenter le nombre de stations modernes de distribution d'essence.

Ces mesures préventives doivent être respectées et renforcées compte tenu du nombre de décès engendrés par les brûlures.

La fréquence des brûlures était plus élevée au cours des mois de décembre, janvier et février, correspondant à la période du harmattan où les températures les plus élevées sont enregistrées coïncidant avec le début de la saison sèche et la pratique des feux de brousse dans notre pays, une des causes de brûlure accidentelle. Iqbal et al. (2011) rapportent que l'hiver est une période à risque de brûlure (63\%). L'utilisation de bains chauds, de buchers domestiques pour se réchauffer pendant cette période expliquerait la survenue de la brûlure. Les données en ce qui concerne la SCB dépendent surtout des circonstances de survenue et de l'agent causal de la brûlure et de son temps d'exposition. Quant aux perturbations biologiques objectivées elles s'expliquent par les conséquences physiopathologiques des brulures, notamment les fuites hydroélectrolytiques et les phénomènes inflammatoires (TNF) et infectieux conduisant à l'hémolyse et par conséquent la constitution d'une anémie (Women's and Children's Hospital - Paediatric Burns Service Guidelines, 2018).

\section{Données thérapeutiques}

La majorité des patients n'avaient rien appliqué sur la plaie sur les lieux de l'accident. Par contre pour Ewono et al. (2014), des produits étaient le plus souvent appliqués; il s'agit du miel, de l'huile, du jaune d'œuf, de la pâte dentifrice. Zahid et al. (2011) quant à eux rapportent 47,3\% de refroidissement à l'eau de robinet, ce qui constitue un bon geste thérapeutique dans la limitation de l'approfondissement des lésions. Dans notre étude, l'antibiothérapie avait été systématiquement instituée contrairement à ce que recommande la littérature (SFETB, 2009 ; Ugburo et al., 2004). Cependant, l'unité de réanimation de l'hôpital universitaire de Parakou est unité de réanimation polyvalente non adaptée à la prise en charge des grands brûlés avec un risque non moins important d'infection nosocomiale plus accru chez le brûlé. Par ailleurs, les raisons de l'absence d'interventions chirurgicales sont multiples allant de l'absence d'un plateau technique adéquat à la mauvaise cicatrisation des plaies en raison de la surinfection, laissant très peu de chance de réussite d'une chirurgie.

\section{Données évolutives et pronostiques}

La mortalité dans notre étude était de $40,8 \%$, très élevée par rapport aux 5,5\% et 21,3\% rapportées respectivement par (Mohammad et al., 2012 ; 
Agbenorku et al., 2013). Nos pays à ressources limitées sont confrontés à des taux de mortalité plus élevés (Peck et al., 2013) liés au plateau technique défaillant et aux conditions de vie précaires de la population. Les complications sont fréquentes chez les brûlés graves. La survenue d'une anémie, d'un choc hypovolémique ou d'un sepsis constitue un facteur de mauvais pronostic comme énoncé dans la littérature.

\section{Conclusion}

Les brûlures graves sont un problème majeur de santé publique. Elles restent très fréquentes chez les enfants en bas âges. Les causes sont multiples et dominées par l'ébouillantement et les flammes. Leur prise en charge est confrontée à de multiples difficultés dans notre structure sanitaire. L'évolution est marquée par la survenue de nombreuses complications responsables de décès précoces voire secondaires. Dans ce sens, une prise en charge multidisciplinaire mieux codifiée serait salutaire. Des efforts restent à consentir afin de diminuer leur incidence et la forte mortalité.

\section{Déclaration d'intérêts:}

Les auteurs déclarent ne pas avoir de conflits d'intérêts en relation avec cet article.

\section{References:}

1. Agbenorku, P., Agbenorku, M., \& Fiifi-Yankson, PK. (2013). Pediatric burns mortality risk factors in a developing country's tertiary burns intensive care unit. Int J Burns Trauma, 3(3):151-158.

2. Behzad, KM. \& Satar, R. (2014). Analyse épidémiologique et coût de l'hospitalisation pédiatrique associée à la brûlure à Kermanshah, Iran. International Journal of Pediatrics, 2 (4.3) : 369-376.

3. Hyder, A., Kashyap, K., Fishman, S., \& Wali, S. (2012). Review of childhood burn injuries in Sub Saharan Africa: A forgotten public challenge. Afr Saf Promot, 2:43-58.

4. Iqbal, T. \& Saaiq, M. (2011). L'enfant brûlé: un profil épidémiologique et les résultats. J Coll Surg médecins Pak, 21 (11): 691-4.

5. Khongwar, D., Hajong, R., Saikia, J., Topno, N., Baruah, AJ., \& Komut, O. (2016). Clinical study of burn patients requiring admission: A single center experience at North Eastern Indira Gandhi Regional Institute of Health and Medical Sciences. J Family Med Prim Care. Apr-Jun; 5(2):444-448.

6. Kouamé, KE., Abhé, CM., Yapo, YP., Koffi, N., Pete, Y., Coulibaly, Y., Vilasco, B., \& Brouh, Y. (2013). Prise en charge des brûlures graves à Abidjan (Côte d'Ivoire). RAMUR, 18 (1): 1-5. 
7. Mohammad, HR., Hamid, RS., Mehrdad, H., Esmaeil, F., \& Mahdi, M. (2012). Épidémiologie des brûlures pédiatriques à Ispahan, en Iran. Arche Trauma Res, 1 (1): 27-30.

8. OMS (2008). Un plan de l'OMS pour la prévention des brûlures et des soins. Genève.

9. Oludiran, OO. \& Umebese, PF. (2009). Pattern and outcome of children admitted for burns in Benin City, mid-western Nigeria. Indian J Plast Surg, 42:189-93.

10. Owono Etoundi, P., Afane Ela, A., Esiéne, A., Ngayap, G., \& Nyemb Nguéné, N. (2014). Facteurs d'allongement du délai d'admission des brulés graves à l'Hôpital Central de Yaoundé. Health Sci. Dis, 15 (3).

11. Pallier, J., Masson, Y., Leclerc, T., \& Lefort, H. (2016). Child and serious acute burn - La brûlure grave de l'enfant. In book: Urgences. Edition: Bialec Chapter: 130 Editors: SFMU - SAMU de France.

12. Peck, M. \& Pressman, MA. (2013). The correlation between burn mortality rates from fire and flame and economic status of countries. Burns, 39: 1054.

13. SFETB (2009). Recommandations on the use of antibiotics in the burned during the acute phase Long text (French Annals of Anesthesia and Intensive Care 28: 265-274.

14. Ugburo, AO., Atoyebi, OA., Oyeneyin, JO., \& Sowemimo, GOA. (2004). An evaluation of the role of systemic antibiotic prophylaxis in the control of burn wound infection at the Lagos University Teaching Hospital. Burns 30: 43-48.

15. Women's and Children's Hospital - Paediatric Burns Service Guidelines (2018). Guidelines for the management for Paediatric Burns.: Updated. p 51.

16. Zahid, A., Atannaz, J., Alaoui, M., Raffik, A., Ezzoubi, M., Diouri, M., Chlihi, A., Bahechar, N., \& Boukind, EH. (2011). Profil épidémiologique des brûlures d'enfants admis au Centre National des Brûlés, Maroc. Ann Burns Fire Disasters, 24 (4) : 171. 